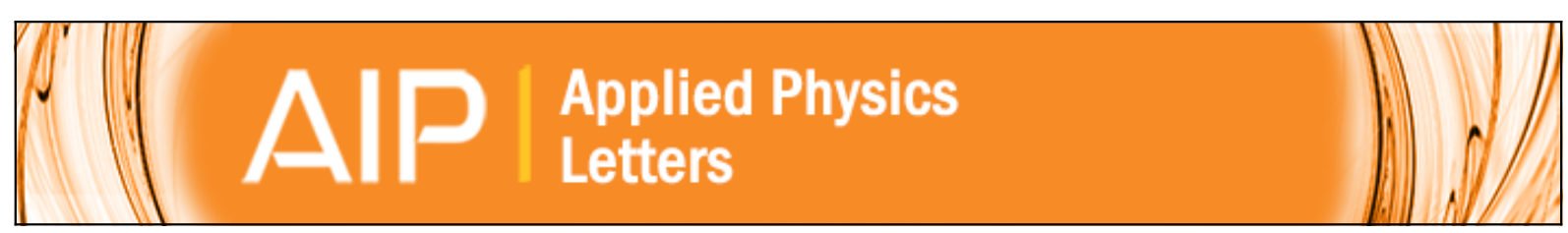

\title{
Thermoelectric properties of heavy-element doped $\mathrm{CrN}$
}

C. X. Quintela, B. Rodríguez-González, and F. Rivadulla

Citation: Applied Physics Letters 104, 022103 (2014); doi: 10.1063/1.4861845

View online: http://dx.doi.org/10.1063/1.4861845

View Table of Contents: http://scitation.aip.org/content/aip/journal/apl/104/2?ver=pdfcov

Published by the AIP Publishing

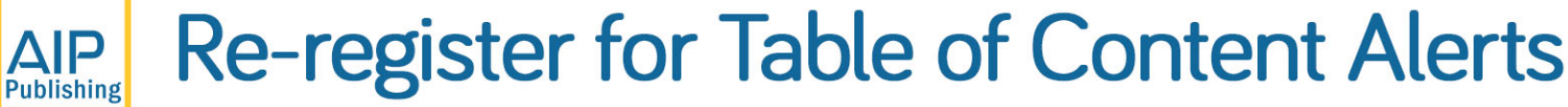

Create a profile.

Sign up today! 


\title{
Thermoelectric properties of heavy-element doped $\mathrm{CrN}$
}

\author{
C. X. Quintela, ${ }^{1}$ B. Rodríguez-González, ${ }^{2}$ and F. Rivadulla ${ }^{1, a)}$ \\ ${ }^{1}$ Centro de Investigación en Química Biológica y Materiales Moleculares (CIQUS), Universidad de Santiago \\ de Compostela, 15782 Santiago de Compostela, Spain \\ ${ }^{2}$ Departamento de Química-Física, Universidad de Vigo, 36310 Vigo, Spain
}

(Received 28 October 2013; accepted 26 December 2013; published online 13 January 2014)

\begin{abstract}
CrN was doped with Mo and W to study the effect of heavy elements alloying on its thermoelectric properties. An spontaneous phase segregation into Mo- and W-rich regions was observed even at the lowest concentrations probed at this work $(\simeq 1 \%)$. In the particular case of $\mathrm{W}$, this segregation creates nanoinclusions into the $\mathrm{Cr}_{1-x} \mathrm{~W}_{x} \mathrm{~N}$ matrix, which results in a substantial reduction of the thermal conductivity in the whole temperature range compared to undoped $\mathrm{CrN}$. In addition, an increased hybridization of $\mathrm{N}: 2 p$ and $4 d / 5 d$ orbitals with respect to Cr:3d decreases the electrical resistivity in lightly doped samples. This improves substantially the thermoelectric figure of merit with respect to the undoped compound, providing a pathway for further improvement of the thermoelectric performance of CrN. @ 2014 AIP Publishing LLC. [http://dx.doi.org/10.1063/1.4861845]
\end{abstract}

The use of thermoelectric generators, which can transform a thermal gradient into usable electricity, have gained importance for clean electricity production from waste heat or natural heat sources. ${ }^{1}$ The conversion efficiency of a thermoelectric material working at a temperature $T$ is related to the thermoelectric figure of merit, $Z=S^{2} \sigma /\left(\kappa_{e}+\kappa_{l}\right)$, where $S$ is the Seebeck coefficient, $\sigma$ is the electrical conductivity, and $\kappa_{e}$ and $\kappa_{l}$ are the electrical and lattice thermal conductivities, respectively. The main difficulty in developing efficient thermoelectric materials is related to the fact that $S, \sigma$, and the total thermal conductivity, $\kappa=\kappa_{e}+\kappa_{l}$, cannot be treated independently; therefore, a compromise solution must be reached when trying to improve $Z$.

The are two standard approaches for the optimization of $Z$ : one is to optimize the power factor, $P F=S^{2} \sigma$, by tuning the electronic properties of the material; the second consists on reducing $\kappa_{l}$ by effectively scattering phonons. ${ }^{1}$ Strategies to increase $P F$ include optimizing the carrier concentration via chemical doping, ${ }^{1}$ introducing resonant impurity levels near the Fermi level ${ }^{2}$ or reducing the dimensionality of the system. ${ }^{3}$ Lowering $\kappa_{l}$ is usually achieved by nanostructuring the material ${ }^{4}$ or introducing defects of different sizes via alloying and/or multiphase segregation. ${ }^{5}$ However, minimizing $\kappa_{l}$ while maintaining large $P F$ is challenging because phonons scatterers also reduce the electronic mobility, which results detrimental to $P F$. An effective way to escape from this handicap is to take advantage of the different mean free paths of electrons and phonons, introducing defects which preferably impede the transport of heat more effectively than the charge.

Following this approach, we thought that doping $\mathrm{CrN}$ at the $\mathrm{Cr}$-site with heavier elements of the same group could reduce the thermal conductivity, at the time that is beneficial to $P F$. A larger hybridization of $4 d$ and $5 d$ orbitals with $\mathrm{N}: 2 p$ could reduce the electronic correlations characteristic of Mott insulator $\mathrm{CrN}^{6}{ }^{6}$ increasing its electronic mobility. Based in such premise, the effect of heavy element doping on the thermoelectric properties of bulk $\mathrm{CrN}$ was studied by substituting $\mathrm{Cr}$ by Mo and W.

\footnotetext{
${ }^{a)}$ Electronic mail: f.rivadulla@usc.es
}

Stoichiometric $\mathrm{CrN}$ is a degenerated semiconductor, which was recently considered as potential thermoelectric material due to its large Seebeck coefficient $\left(-135 \mu \mathrm{V} \mathrm{K}^{-1}\right.$ at $\left.300 \mathrm{~K}\right)$ and its rather low thermal conductivity $\left(1.76 \mathrm{~W} \mathrm{~m}^{-1} \mathrm{~K}^{-1}\right.$ at $300 \mathrm{~K}) .^{7}$ The material shows good mechanical properties, including large hardness, high-temperature stability, strong adhesion to different surfaces, and excellent oxidation and wear resistance. ${ }^{8,9}$ At $T_{N} \approx 273-286 \mathrm{~K}, \mathrm{CrN}$ undergoes a magnetostructural phase transition (MPT) from a high-temperature paramagnetic (PM) cubic $\mathrm{Fm} \overline{3} m$ phase to a low-temperature antiferromagnetic (AFM) orthorhombic Pnma phase, ${ }^{10}$ associated to a large exchange-striction. ${ }^{11,12}$ Its $\kappa(T)$ is also puzzling: while in the low temperature AFM phase $\kappa(T)$ is fairly consistent with the expectation for a crystalline material, in the high temperature PM phase $\kappa$ shows an unexpected linear temperature dependence. This behavior, distinctive of amorphous materials, is not expected at all in the cubic phase of $\mathrm{CrN}$, where the lattice contribution to the thermal conductivity is the dominant.

Polycrystalline $\mathrm{Cr}_{1-x} \mathrm{~W}_{x} \mathrm{~N}$ with $x=(0.01$ and 0.02$)$ and $\mathrm{Cr}_{1-x} \mathrm{Mo}_{x} \mathrm{~N}$ with $x=(0.01,0.025,0.05,0.1)$ were synthesized as described in Ref. 13. Structural and morphological characterization of the samples was addressed by powder $\mathrm{x}$-ray diffraction (XRD), high-resolution transmission electron microscopy (TEM), and energy dispersion spectroscopy (EDS). Electrical resistivity measurements were performed by using the four-contact configuration on thin bars cut from dense pellets. Thermal conductivity was measured by using the steady-state absolute method on dense bars of $6 \mathrm{~mm}$ length and a cross sectional area of $2 \mathrm{~mm} \times 1 \mathrm{~mm}$. Seebeck coefficient was measured applying a heat pulse on one of the ends of the sample and recording the $\Delta V / \Delta T$ variation at each temperature. The three magnitudes entering $Z$ were measured on the same sample for each composition.

The XRD patterns of the different samples are shown in Fig. 1. For all the $\mathrm{Cr}_{1-x} \mathrm{Mo}_{x} \mathrm{~N}$ samples with $x \geq 0.025$, there were detected low intensity diffraction peaks associated to hexagonal MoN structure $\left(\delta_{3}\right)$ (space group $P 6_{3} m c$ ). As shown in Fig. 1(b), these impurity peaks move toward the theoretical position of hexagonal MoN (200) as the Mo concentration increases. In the case of the $\mathrm{W}$-doped samples, we 


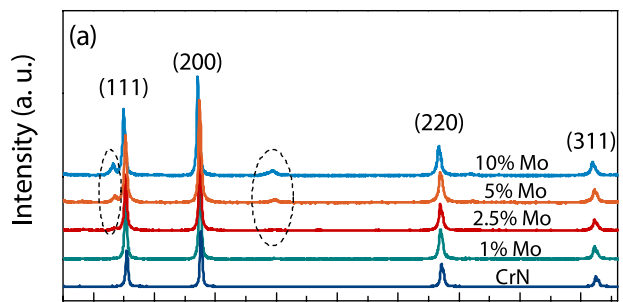

$\begin{array}{lllllllll}35 & 40 & 45 & 50 & 55 & 60 & 65 & 70 & 75\end{array}$
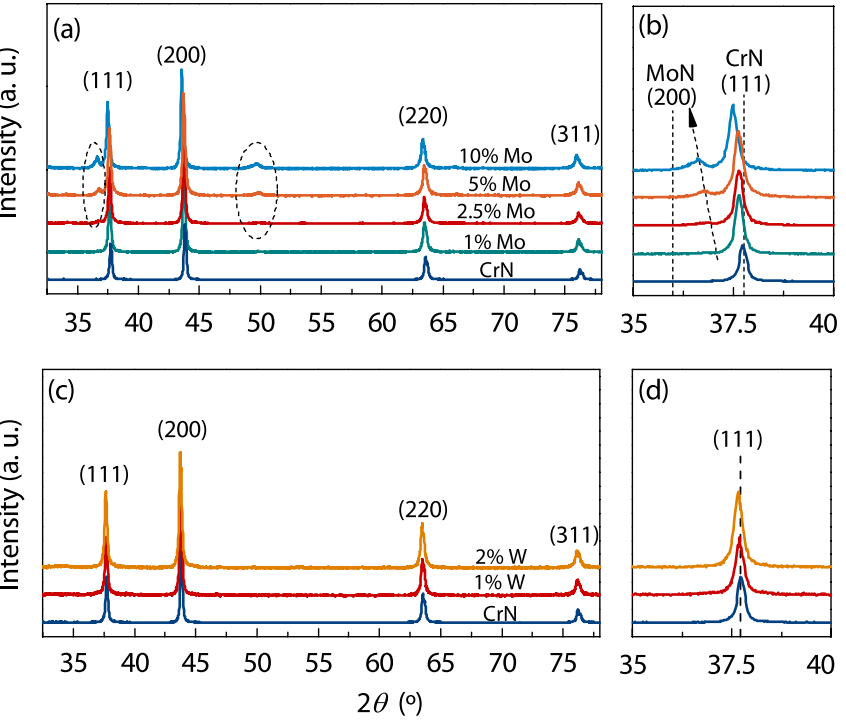

FIG. 1. (a) XRD patterns for the series $\mathrm{Cr}_{1-x} \mathrm{Mo}_{x} \mathrm{~N}$. Dashed ovals show diffraction peaks associated to hexagonal MoN. (b) Zoom in the vicinity of the $\mathrm{CrN}$ (111) peak for the members of the $\mathrm{Cr}_{1-x} \mathrm{Mo}_{x} \mathrm{~N}$ series. The arrow shows the presence of the (002) peak of hexagonal MoN above 2.5\% Mo. (c) XRD patterns of the $\mathrm{Cr}_{1-x} \mathrm{~W}_{x} \mathrm{~N}$ series. (d) Zoom in the vicinity of the $\mathrm{CrN}$ (111) peak showing the absence of impurity peaks at this small level of doping with $\mathrm{W}$.

have kept the $\mathrm{W}$ concentration below the $2.5 \%$ limit to avoid a similar effect of segregation. In this case, only the peaks corresponding to cubic $\mathrm{CrN}$ are observed, indicating the absence of secondary phases. However, it is important to note that, both in Mo and W samples, the diffraction peaks characteristic of $\mathrm{CrN}$ shift to lower angles, indicating an increase in the lattice parameter with respect to the undoped compound. This suggests a successful replacement of part of $\mathrm{Cr}$ atoms by $\mathrm{Mo}$ and $\mathrm{W}$ in the rock-salt structure.

In order to further analyze the distribution of dopants and the effect on the morphology of the CrN crystals, we have performed high-resolution TEM characterization and EDS analysis of the samples (Fig. 2). The analysis reveals that the material forms a solid solution in a very limited range of compositions, and it segregates spontaneously even for substitutions as small as 1\% of W/Mo. Therefore, even for samples with clean XRD patterns, there is a nanoscale segregation of dopant ions. In the case of W-doped samples, three levels of doping can be differentiated from the analysis of XRD and TEM: a small amount of $\mathrm{W}$ replaces $\mathrm{Cr}$ in the host $\mathrm{CrN}$ lattice forming a true alloy, as reflected in the progressive increase of the cubic lattice parameter. As shown in Fig. 2, further doping results in segregation of W-rich regions within the $\mathrm{CrN}$ lattice. Even further doping results in a complete segregation of WN particles. For Mo, there is a larger tendency towards complete segregation of MoN particles even for the lowest doping. ${ }^{14}$

The effect of atomic replacement combined with the compositional phase segregation is reflected in the evolution of the electrical resistivity with doping. As shown in Figs. 3(a) and 3(b), the value of the electrical resistivity in the whole temperature range decreases with respect to stoichiometric CrN. Hall effect measurements performed in $\mathrm{Cr}_{0.92} \mathrm{~W}_{0.02} \mathrm{~N}$ showed a room temperature carrier concentration of $n \approx 2.7 \times 10^{19} \mathrm{~cm}^{-3}$, comparable to the observed in pure $\mathrm{CrN}\left(n \approx 4.1 \times 10^{19} \mathrm{~cm}^{-3}\right)$. This confirms that the

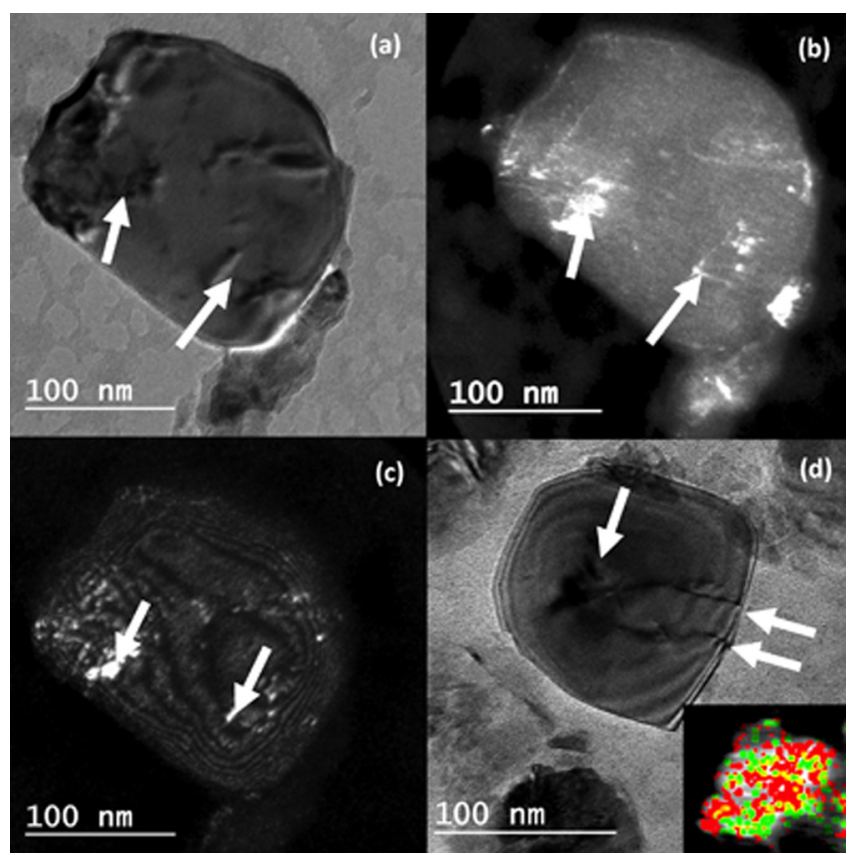

FIG. 2. Bright field ((a) and (d)) and dark field ((b) and (c)) TEM images of $2 \% \mathrm{~W}: \mathrm{CrN}$ particles. The arrows mark crystalline defects that could be due to compositional variations across the particles. The inset in panel (d) presents the EDS mapping of $\mathrm{W}$ (red) and $\mathrm{Cr}$ (green) in a single $\mathrm{Cr}_{0.98} \mathrm{~W}_{0.02} \mathrm{~N}$ particle.

decrease in the resistivity is due to a larger mobility in a wider, less correlated band due to an increase of the M: $d$ - $\mathrm{N}: 2 p$ hybridization for $4 d$ and $5 d$ metals.

The lower resistivity is achieved for $\mathrm{Cr}_{0.99} \mathrm{Mo}_{0.01} \mathrm{~N}$ and $\mathrm{Cr}_{0.99} \mathrm{~W}_{0.01} \mathrm{~N}$, and it increases gradually with doping beyond this level. This suggests that the solubility limit must be around 1\% W/Mo concentration. Therefore, the segregation of (W/Mo)-rich regions scatter electrons, increasing the electrical resistivity for concentrations larger than $1 \%$. It should be also mentioned that for the most conductive samples, although still thermally activated, the low temperature resistivity does not diverge at all, suggesting its closeness to an itinerant electron regime.

The temperature dependence of the Seebeck coefficient is shown in Figs. 3(c) and 3(d). All the samples exhibit negative values of $S$ in the whole temperature range, pointing that the majority carriers are electrons, in agreement to Hall effect measurements. Comparing the resistivity and Seebeck coefficient, it can be concluded that the decrease in the absolute value of Seebeck coefficient (in comparison with pure $\mathrm{CrN}$ ) is consistent with the decrease observed in the electrical resistivity. Moreover, the Seebeck coefficient continues to increase linearly, without signs of saturation at high temperature, for the samples with the smaller resistivity $(\simeq 1 \%)$. As a consequence, the $P F$ increases at $\simeq 1 \%(\mathrm{~W} / \mathrm{Mo}): \mathrm{CrN}$, doubling the value reported for $\mathrm{CrN}$ at $400 \mathrm{~K}^{7}$

The temperature dependence of the thermal conductivity for $\mathrm{CrN}$ and for the Mo- and $\mathrm{W}$-doped compounds is shown in Figs. 3(e) and 3(f). Pure CrN shows a broad maximum, with a very small temperature dependence of $\kappa$ from $\simeq 125-250 \mathrm{~K}$. Above this temperature, $\kappa(T)$ decreases abruptly due to the magnetostructural transition $\left(\mathrm{T}_{N} \approx\right.$ $285 \mathrm{~K}$ ) and increases linearly in the PM cubic phase above $\mathrm{T}_{N}{ }^{7}$ The effect of Mo and $\mathrm{W}$ on $\kappa(T)$ is very different for 

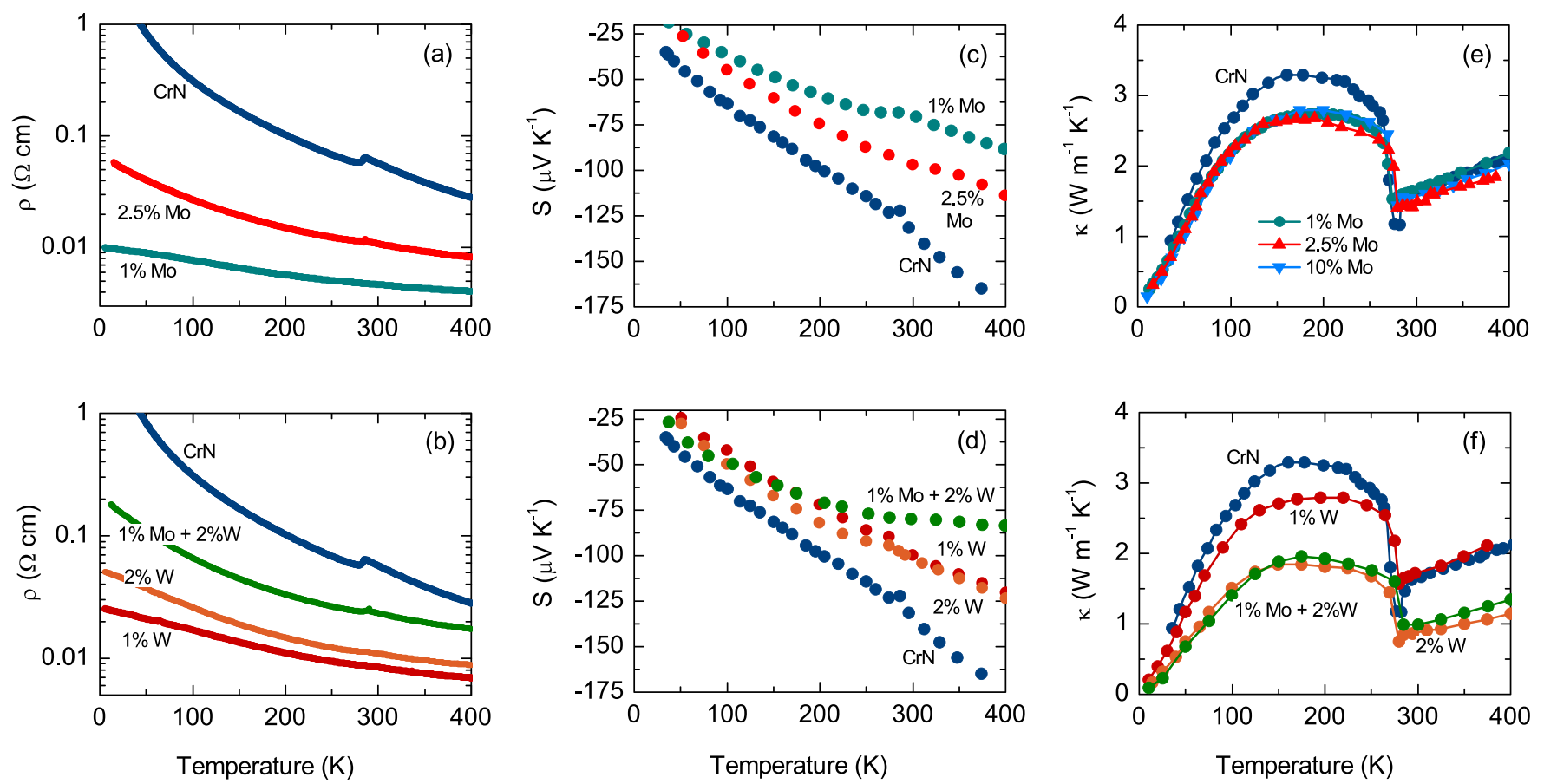

FIG. 3. Temperature dependence of $\rho((a)$ and (b)), $S((\mathrm{c})$ and (d)), and $\kappa((\mathrm{e})$ and (f)), for the most representative samples studied in this work.

both ions: $1 \%$ of either Mo or $\mathrm{W}$ reduce slightly the thermal conductivity below $\mathrm{T}_{N}$, but leave $\kappa$ the same as in pure $\mathrm{CrN}$, above $\mathrm{T}_{N}$. However, while further doping with Mo (up to $10 \%$ ) does not reduce $\kappa, 2 \%$ of $\mathrm{W}$ decreases further the thermal conductivity in the whole temperature range. We have also co-doped $\mathrm{CrN}$ with $1 \% \mathrm{Mo}+2 \% \mathrm{~W}$ but the result is identical to that observed for $2 \% \mathrm{~W}$, showing the dominant effect of $\mathrm{W}$ on the reduction of $\kappa(T)$. Therefore, it can be concluded from these data that higher levels of doping will result in a complete segregation of (W/Mo)N particles, which will not reduce further the lattice thermal conductivity of $\mathrm{CrN}$.

The effect of the different scattering mechanisms that produce a change on $\kappa_{l}$ is usually studied using the Boltzmann transport equation for phonons. In a first approximation, $\kappa_{l}$ can be estimated by the Debye model according to ${ }^{15}$

$$
\kappa_{l}(T)=\frac{1}{2 \pi^{2} v} \int_{0}^{\omega_{D}} \tau(\omega) \frac{\hbar^{2} \omega^{4}}{k_{B} T^{2}} \frac{e^{\frac{\hbar \omega}{k_{B} T}}}{\left(e^{\frac{\hbar \omega}{k^{T}}}-1\right)^{2}} d \omega
$$

where the relaxation time, $\tau(\omega)$, is the sum of the contribution of different processes that produce an effective phonon scattering. In this study, the combined relaxation time using the Matthiessen's rule is given as ${ }^{16}$

$$
\tau^{-1}(\omega)=\frac{v}{1.8 d}+\frac{\hbar \gamma^{2}}{M v^{2} \theta_{D}} \omega^{2} T e^{\frac{-\theta_{D}}{\alpha T}}+C \omega^{r} .
$$

Here, the first term represents the phonon-boundary scattering, which depends on the sound velocity of the material, $v$, and the grain size, $d$. The second term represents the phonon-phonon Umkalpp scattering processes, which depends on the average atomic mass in the crystal, $M$, the Grüneissen constant, $\gamma$, the Debye temperature, $\theta_{D}$, and a empirical constant, $\alpha$. The last term takes into account the scattering of phonons due to point defects in the lattice. For point defects or any other object with a typical size much smaller than the phonon wavelength, this term reduces to Rayleigh scattering, having $r=4$ and

$$
C=\frac{V_{0}}{4 \pi v^{3}} \sum_{i} x_{i} N_{i}^{2}
$$

where $V_{0}$ and $x_{i}$ are the volume and molar fraction of defects, and $N$ represents the contributions of the local variation of mass and bond distance due to the defects on the host lattice. ${ }^{17}$ Joshi $^{18}$ proved that when the size of the defects is comparable to the phonon-wavelength, the objects cannot be considered as point defects and $r=2$ must be considered.

In order to get an insight on the physical mechanism that produces the observed reduction of the thermal conductivity in the $\mathrm{W}$-doped samples, we have fitted the experimental data below $\mathrm{T}_{N}$ to Eq. (1), considering the different contributions to the relaxation time according to Eq. (2). The fittings are shown in Fig. 4. We have calculated the contribution of the Umklapp term using $\theta_{D}=460 \mathrm{~K}$ and $v=5575 \mathrm{~m} / \mathrm{s}$ (from specific heat measurements), and $\gamma=2$. These values were kept constant in the calculations for three samples. Fitting the curves that way allows us to discriminate the effect of defect scattering as the concentration of $\mathrm{W}$ increases. Using the nominal \% of $\mathrm{W}$, the fittings reveal a decrease of the phonon mean free path and an increase of the volume of the scattering defects, $V_{0}$, as the amount of $\mathrm{W}$ increases. The $V_{0}$ value obtained for $\mathrm{CrN}\left(V_{0} \approx\right.$ $\left.5 \times 10^{-3} \mathrm{~nm}^{-3}\right)$ is compatible with the volume expected for point defects of effective radius $r_{\text {eff }} \approx 1 \AA$. As the $\mathrm{W}$ concentration is increased, the volume of the scattering defects increases from $V_{0} \approx 7.9 \times 10^{-2} \mathrm{~nm}^{-3}$ for $\mathrm{Cr}_{0.99} \mathrm{~W}_{0.01} \mathrm{~N}$ to $V_{0} \approx 8.9 \times 10^{-2} \mathrm{~nm}^{-3}$ for $\mathrm{Cr}_{0.98} \mathrm{~W}_{0.02} \mathrm{~N}$. Consonant with these results, the parameter $d$ (associated to the grain size) decreases from $d \approx 13 \mathrm{~nm}$ for $\mathrm{CrN}$, to $d \approx 10 \mathrm{~nm}$ for $\mathrm{Cr}_{0.99} \mathrm{~W}_{0.01} \mathrm{~N}$, and $d \approx 7.5 \mathrm{~nm}$ for $\mathrm{Cr}_{0.98} \mathrm{~W}_{0.02} \mathrm{~N}$. Note that a reduction in the effective doping of $\mathrm{W}$ (due to segregation of 


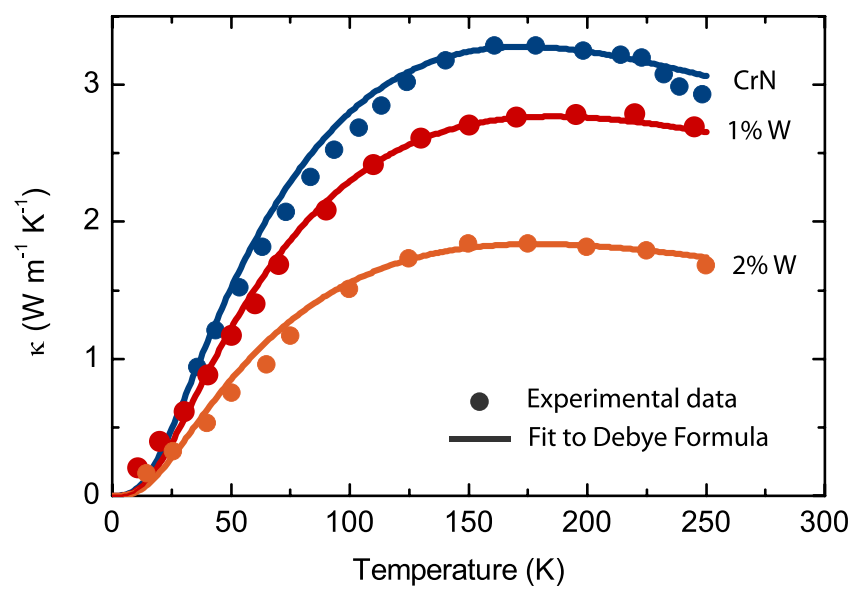

FIG. 4. Temperature dependence of $\kappa$ for $\mathrm{CrN}, \mathrm{Cr}_{0.98} \mathrm{~W}_{0.02} \mathrm{~N}(2 \% \mathrm{~W})$, and $\mathrm{Cr}_{0.99} \mathrm{~W}_{0.01} \mathrm{~N}(1 \% \mathrm{~W})$ and their corresponding fits according to Eq. (1).

WN) will require a larger volume of the scattering defects and, therefore, a smaller average grain size.

The effect of the nanoinclusions seem also very effective in reducing $\kappa(T)$ in the high-temperature PM cubic phase. As we explained before, the linear temperature dependence of $\kappa$ in this phase is characteristic of amorphous materials, instead of a highly crystalline material like this. This temperature dependence cannot be attributed to $\kappa_{e}$ because its value, obtained from the Wiedemann-Franz law, is very small $\left(\kappa_{e} \approx 0.07 \mathrm{Wm}^{-1} \mathrm{~K}^{-1}\right)$, pointing that $\kappa$ is dominated by $\kappa_{l}$. Moreover, in the case of the $2 \% \mathrm{~W}$ sample, the actual value of $\kappa$ above $\mathrm{T}_{N}$ decreases with respect to $\mathrm{CrN}$, in spite of its higher electrical conductivity. Therefore, the linear behavior of $\kappa$ in the high-temperature PM cubic phase is dominated by the lattice thermal conductivity.

In a situation in which defects are not small compared to the phonon wavelength, the influence of Umklapp scattering could be largely suppressed and Rayleigh dispersion mechanism is no longer applicable. Instead, Joshi ${ }^{18}$ proposed a $\tau^{-1}=C \omega^{2}$, which results in a linear $\kappa(T)$ from Eq. (1). Therefore, the reduction of the thermal conductivity above $\mathrm{T}_{N}$ produced by $\mathrm{W}$-doping could be due to the existence of large (nanometer) size inclusions in the $\mathrm{CrN}$ matrix. On the other hand, the inefficacy of Mo to reduce $\kappa_{l}$ in $\mathrm{CrN}$ as effectively as W must be related to the tendency towards segregation of Mo and not due to the formation of nanoinclusions, as observed by XRD and TEM (see supplementary material for TEM images of the Mo-doped samples).

This experiment also provides a valuable insight into the origin of the linear term of $\kappa_{l}(T)$ in the PM cubic phase of $\mathrm{CrN}$. The strong exchange-striction interaction responsible of the magneto-structural transition in $\mathrm{CrN}$ may introduce dynamic bond-length fluctuations that result in an inhomogeneous medium for the propagation of phonons. This effect has been observed in oxides close to a localized-to-itinerant electronic transition, ${ }^{19}$ as it could be the case here.

From the experimental values of $\rho(T), S(T)$, and $\kappa(T)$, the thermoelectric power factor and the thermoelectric figure of merit were calculated. Fig. 5 shows the temperature dependence of $P F$ and $Z T$ for those samples with best thermoelectric performance. Among all the members of the $\mathrm{Cr}_{1-x} \mathrm{~A}_{x} \mathrm{~N}$ series, those with $x=0.01$, show the largest $P F$.

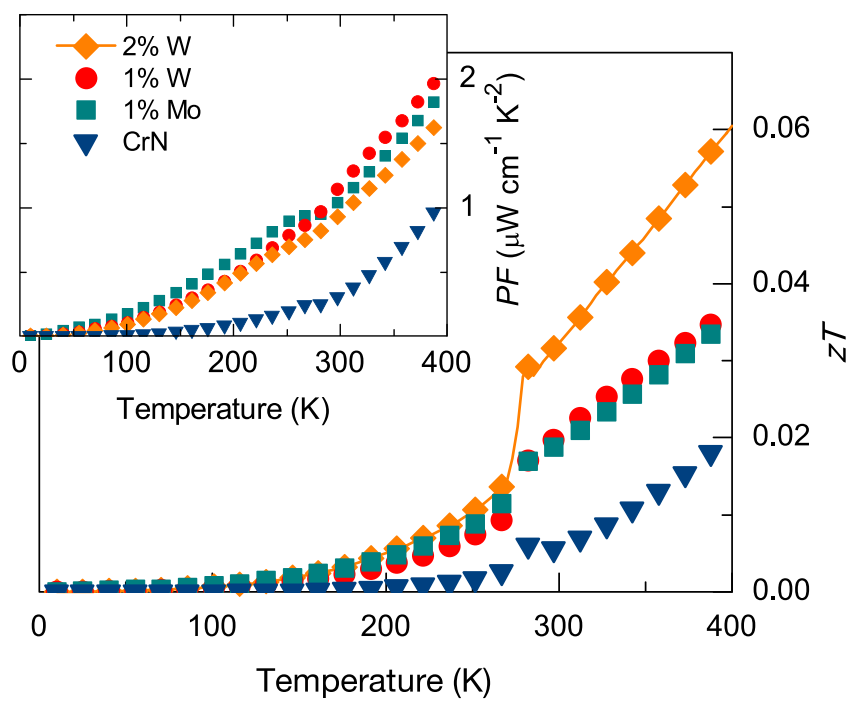

FIG. 5. Temperature dependence of $P F$ and $Z T$ for $\mathrm{Cr}_{0.98} \mathrm{~W}_{0.02} \mathrm{~N}(2 \% \mathrm{~W})$, $\mathrm{Cr}_{0.99} \mathrm{~W}_{0.01} \mathrm{~N}(1 \% \mathrm{~W})$, and $\mathrm{Cr}_{0.99} \mathrm{Mo}_{0.01} \mathrm{~N}(1 \% \mathrm{Mo})$.

The maximum $P F$ is obtained for $\mathrm{Cr}_{0.99} \mathrm{~W}_{0.01} \mathrm{~N}$, which reaches $P F \approx 2 \mu \mathrm{W} \mathrm{cm}{ }^{-1} \mathrm{~K}^{-2}$ at $400 \mathrm{~K}$. This value is roughly two times larger than the observed in stoichiometric CrN. On the other hand, $\mathrm{Cr}_{0.98} \mathrm{~W}_{0.02} \mathrm{~N}$ shows the largest $Z T$, reaching a value of $Z T=0.06$ at $400 \mathrm{~K}$. It is also important to point that in the case of $\mathrm{Cr}_{0.98} \mathrm{~W}_{0.02} \mathrm{~N}, Z T$ increases very rapid with temperature, with a tendency to further enhancement at higher temperatures.

In conclusion, the strong reduction of the thermal conductivity of $\mathrm{CrN}$ produced by doping with $\mathrm{W}$ is due to the presence of $\mathrm{W}$-rich nanoinclusions which scatter phonons very effectively. In the high-temperature PM cubic phase, the presence of these large objects also affects the thermal conductivity, giving an insight about the origin of this intriguing linear temperature dependence of $\kappa(T)$ in $\mathrm{CrN}$. The increased hybridization between $\mathrm{N}: 2 p$ and $4 d / 5 d$ orbitals with respect to $\mathrm{Cr}: 3 d$ decreases the electronic resistivity, resulting in an increase of $P F$. As a result, the thermoelectric figure of merit is substantially increased with respect to pure $\mathrm{CrN}$. Note that the $P F$ of $\mathrm{Cr}_{0.9} \mathrm{~V}_{0.1} \mathrm{~N}$ was previously reported to be $P F=4 \mu \mathrm{W} \mathrm{cm}{ }^{-1} \mathrm{~K}^{-2}$ at $400 \mathrm{~K}$ due to the optimization of charge carrier concentration. ${ }^{7}$ If this is combined with the reduction of the thermal conductivity achieved for $\mathrm{Cr}_{0.98} \mathrm{~W}_{0.02} \mathrm{~N}$, it would lead to $Z T \approx 0.1$ at room temperature, suggesting that co-doping strategies could improve the high temperature performance of $\mathrm{CrN}$.

This work was supported by the European Research Council (ERC StG-259082, 2DTHERMS), Xunta de Galicia (Project No. 2012-CP071), and by the Ministry of Economy and Competitiveness (Project No. MAT2010-16157).

${ }^{1}$ F. J. Di Salvo, Science 285, 703 (1999).

${ }^{2}$ J. P. Heremans, B. Wiendlocha, and A. M. Chamoire, Energy Environ. Sci. 5, 5510 (2012).

${ }^{3}$ J. Sootsman, H. Kong, C. Uher, J. D’Angelo, C.-I. Wu, T. Hogan, T. Caillat, and M. Kanatzidis, Angew. Chem., Int. Ed. 47, 8618 (2008).

${ }^{4}$ M. Dresselhaus, G. Chen, M. Tang, R. Yang, H. Lee, D. Wang, Z. Ren, J.-P. Fleurial, and P. Gogna, Adv. Mater. 19, 1043 (2007).

${ }^{5}$ S.-H. Lo, J. He, K. Biswas, M. G. Kanatzidis, and V. P. Dravid, Adv. Funct. Mater. 22, 5175 (2012). 
${ }^{6}$ A. S. Botana, F. Tran, V. Pardo, D. Baldomir, and P. Blaha, Phys. Rev. B 85, 235118 (2012).

${ }^{7}$ C. X. Quintela, F. Rivadulla, and J. Rivas, Appl. Phys. Lett. 94, 152103 (2009).

${ }^{8}$ Y. Otani and S. Hofmann, Thin Solid Films 287, 188 (1996).

${ }^{9}$ J. Lin, B. Mishra, J. Moore, and W. Sproul, Surf. Coat. Technol. 202, 3272 (2008).

${ }^{10}$ L. M. Corliss, N. Elliott, and J. M. Hastings, Phys. Rev. 117, 929 (1960).

${ }^{11}$ A. Filippetti and N. A. Hill, Phys. Rev. Lett. 85, 5166 (2000).

${ }^{12}$ F. Rivadulla, M. Banobre-Lopez, C. X. Quintela, A. Pineiro, V. Pardo, D. Baldomir, M. A. Lopez-Quintela, J. Rivas, C. A. Ramos, H. Salva, J.-S. Zhou, and J. B. Goodenough, Nature Mater. 8, 947 (2009).
${ }^{13}$ C. X. Quintela, F. Rivadulla, and J. Rivas, Phys. Rev. B 82, 245201 (2010).

${ }^{14}$ See supplementary material at http://dx.doi.org/10.1063/1.4861845 for TEM images and EDS microanalysis mappings of the Mo- and W-doped samples.

${ }^{15}$ J. Callaway, Phys. Rev. 113, 1046 (1959).

${ }^{16}$ Thermal Conductivity: Theory, Properties and Applications, Physics of Solids and Liquids, edited by T. Tritt (Springer, 2005).

${ }^{17}$ C. T. Walker and R. O. Pohl, Phys. Rev. 131, 1433 (1963).

${ }^{18}$ Y. P. Joshi, Phys. Status Solidi B 95, 627 (1979).

${ }^{19}$ B. Rivas-Murias, H. D. Zhou, J. Rivas, and F. Rivadulla, Phys. Rev. B 83, 165131 (2011). 